\title{
Spin Lifetime in Electron-Doped InP Quantum Dots
}

\author{
Y. Masumoto*, B. Pal*, S. Oguchi* and M. Ikezawa* \\ *Institute of Physics, University of Tsukuba, Tsukuba 305-8571, Japan
}

\begin{abstract}
Spin relaxation of electrons doped in InP quantum dots was studied by means of luminescence pump-probe and Hanle measurements. Optical pumping makes spins of doped electrons to be oriented in parallel to the helicity of the circularly polarized excitation. The luminescence pump-probe showed the spin orientation of the doped electrons decay on a millisecond time-scale. Hanle measurement clarified the spin dephasing relaxation time of doped electrons is $1.7 \mathrm{~ns}$ which is explained by the frozen fluctuation of nuclear spins.
\end{abstract}

Electron spins in quantum dots (QDs) are good candidates for the quantum memory in the quantum information technology, because transferring the quantum information from the photon polarization to the electron spin polarization is direct and one-to-one. Electrons in QDs are expected to have long spin lifetime and doped electrons in QDs have infinite lifetime. We investigated time-resolved optical orientation of charge tunable InP QDs and found that the spins of the doped electrons are oriented under the circularly polarized excitation and are preserved in part for up to sub-millisecond [1]. In this work, we report nanosecond dephasing time of the spins by means of Hanle measurement as well as up to millisecond preservation of the spin by means of photoluminescence (PL) pump-probe in the same QDs.

The samples studied are charge tunable one-layer InP self-assembled QDs grown on $n^{+}$-GaAs substrates. The excitation source was a cw Ti:sapphire laser and the PL was detected by a photomultiplier with a 2-channel gated photon counter (GPC). A photoelastic modulator (PEM) was used to produce right and left circular polarization alternatively at $42 \mathrm{kHz}$ in the detection path or the excitation path. The number of electrons in the QDs was varied with the change of the electric bias applied across the quantum structure. Trionic quantum beats in PL showed that each InP QD contains one doped electron on an average at the electric bias of $-0.1 \sim-0.2 \mathrm{~V}$ [2].

Under the electric bias of $-0.1 \mathrm{~V}$, the negative circular polarization (NCP) was observed in PL of InP QDs under the circularly polarized quasi-resonant excitation and the longitudinal magnetic field of 0.1T. Time-resolved study of the PL circular polarization showed that NCP started at $100 \mathrm{ps}$ and is preserved within the recombination lifetime [1]. The NCP is explained by considering the optical pumping of the spins of doped electrons under the circularly polarized excitation and the simultaneous spin

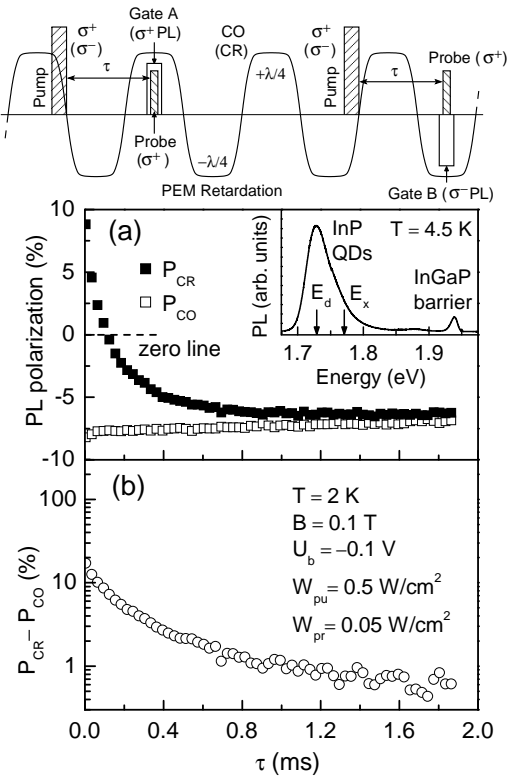

FIGURE 1. (Upper Sketch): Synchronization of retardation in a PEM, probe pulses, and gates of a GPC. (Main Panel): Probe PL polarization for co- $\left(P_{\mathrm{CO}}\right)$ and cross- $\left(P_{\mathrm{CR}}\right)$ circularly polarized pump-probe (a), and the difference $P_{\mathrm{CR}}-P_{\mathrm{CO}}$ vs. pump-probe delay $(\tau)(\mathbf{b})$. PL spectrum is shown in the inset.

flip-flop process of a photogenerated electron-hole pair in P-QDs in which the spin of the doped electron is oriented in parallel to the helicity of the circularly polarized excitation $[1,3]$. For the time-resolved optical orientation of doped electrons in the time region far beyond the PL lifetime, a pump-probe PL was used [3].

The timing of the pump, probe pulses and the gate of the photon counter is shown at the upper part of Fig.1. We measured the probe PL polarization for co-circularly polarized pump-probe $\left(P_{\mathrm{CO}}\right)$ and cross-circularly polar- 

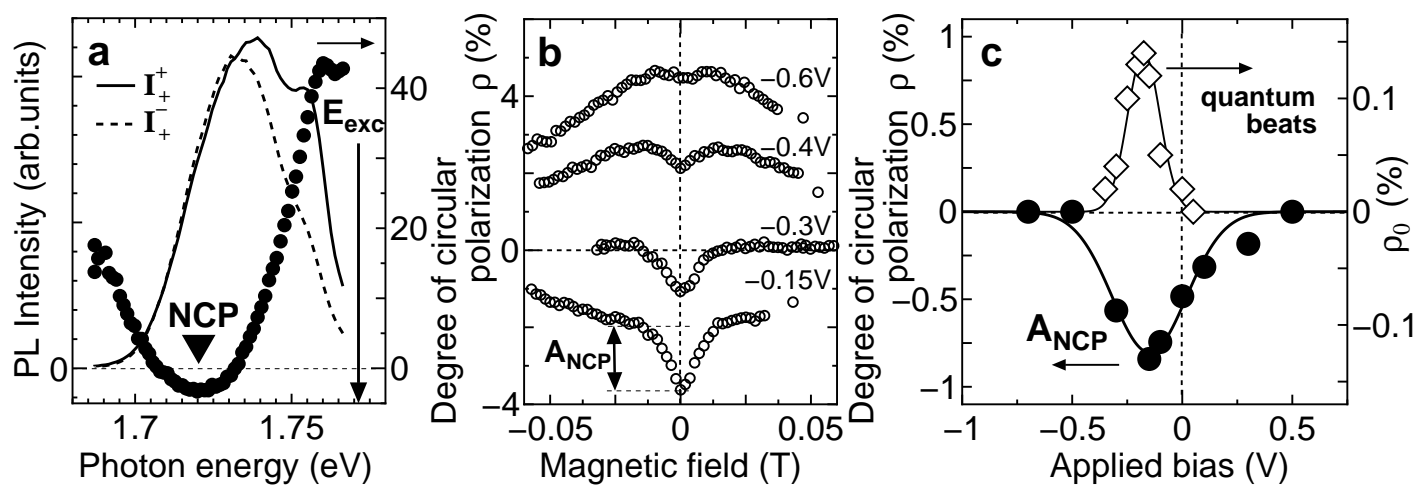

FIGURE 2. a. Circularly polarized PL spectra and circular polarization spectrum of InP QDs. b. PL circular polarization of the electrically biased InP QDs under the transverse magnetic field (Hanle curve). c. Amplitude of the sharp Hanle dip and that of trionic quantum beat as a function of applied electric bias.

ized pump-probe $\left(P_{\mathrm{CR}}\right)$ as a function of pump-probe time delay $\tau$. The experimental data are shown in the main panel of Fig.1. The difference $P_{\mathrm{CR}}-P_{\mathrm{CO}}$ is a good measure of the pump induced spin orientation of the doped electrons. A semi-logarithmic plot of $P_{\mathrm{CR}}-P_{\mathrm{CO}}$ shows that the spin orientation of doped electrons decays nonexponentially and that the spin polarization decays on a millisecond time-scale. Spin relaxation rate increases with the increase of the temperature and the longitudinal magnetic field. The temperature dependence suggests two-phonon processes as the dominant spin relaxation mechanism in QDs at the elevated temperatures.

Under quasi-resonant excitation, PL of singly electron doped InP QDs showed NCP at the Stokes shift of $49 \mathrm{meV}$ even under zero magnetic field, as shown in Fig.2a. We measured the Hanle effect of the sample under the transverse magnetic field. The Hanle curves of singly electron doped InP QDs taken in the magnetic field up to $6 \mathrm{~T}$ and under the electric bias of $-0.1 \mathrm{~V}$ are described by the expression, $\rho(B)=A_{0}+A_{\mathrm{NCP}} /[1+$ $\left.\left(B / B_{1}\right)^{2}\right]+A_{2} /\left[1+\left(B / B_{2}\right)^{2}\right]+A_{3} /\left[1+\left(B / B_{3}\right)^{2}\right]$, where $B_{i}=\hbar / g_{i} \mu_{\mathrm{B}} T_{2, i}^{*},(i=1,2,3)$, and $T_{2, i}^{*}$ is the spin lifetime. Fitting parameters are, $A_{0}=0.10 \%, A_{\mathrm{NCP}}=-1.43 \%$, $B_{1}=4.5 \mathrm{mT}, A_{2}=-3.97 \%, B_{2}=128 \mathrm{mT}, A_{3}=2.30 \%$, and $B_{3}=1.54 \mathrm{~T}$. Lorentzians having half width at the half maximum (HWHM) of $B_{2}$ and $B_{3}$ are observed in neutral InP QDs and are assigned to depolarization of excitons and holes, respectively [4]. The Hanle curves precisely measured in the low field regime up to $62.5 \mathrm{mT}$ are displayed in Figs.2b. Simultaneously with the enhancement of the trionic quantum beat, a sharp dip appeared on a broader Lorentzian in the Hanle curve and the amplitude of the sharp dip of negative circular polarization, $A_{\mathrm{NCP}}$, is enhanced around the applied bias of $-0.2 \mathrm{~V}$, as is shown in Figs.2b and 2c. The clear coincidence between NCP and trionic quantum beat shows sharp Hanle dip is reflected by the long spin dephasing time of doped elec- trons in QDs. The sharp Lorentzian dip has HWHM of $4.5 \mathrm{mT}$. The HWHM of $B_{1}=4.5 \mathrm{mT}$ of the sharp Hanle dip corresponds to the electron spin coherence time of $\tau$ $=1.7 \mathrm{~ns}$, because electron g-factor in InP QDs is 1.5. This observation clearly indicates the spin coherence time of doped electrons in InP QDs is $1.7 \mathrm{~ns}$. The recombination lifetime in InP QDs is 250 ps by the time-resolved PL measurement [1]. The $1.7 \mathrm{~ns}$ spin dephasing time of the doped electron is much longer than the recombination lifetime. The time is almost consistent with the estimated electron spin dephasing time in the randomly distributed frozen fluctuation of the nuclear hyperfine field [5]. It is almost consistent with the dispersion of the hyperfine field caused by nuclear spin fluctuation measured from a clear zero-field dip (HWHM $=15 \mathrm{mT})$ in the plot of the circular polarization vs. the longitudinal magnetic field. Dephasing rate increases with the increase of temperature, and its temperature dependence suggests the twophonon process for dephasing of the electron spin.

This work was supported by the Grant-in-Aid for the Scientific Research \#13852003 and \#18204028 from the MEXT and "R\&D promotion scheme funding international joint research" promoted by NICT of Japan.

\section{REFERENCES}

1. M. Ikezawa, B. Pal, Y. Masumoto, I. V. Ignatiev, S. V. Verbin, and I. Ya. Gerlovin, Phys. Rev. B 72, 153302-1-4 (2005).

2. I. E. Kozin, V. G. Davydov, I. V. Ignatiev, A. V. Kavokin, K. V. Kavokin, G. Malpuech, H. -W. Ren, M. Sugisaki, S. Sugou, and Y. Masumoto, Phys. Rev. B 65, 241312(R)1-4 (2002).

3. B. Pal, M. Ikezawa, Y. Masumoto, and I. V. Ignatiev, $J$. Phys. Soc. Jpn. 75, 54702-1-5 (2006).

4. Multicomponent Hanle curves for electrons and excitons were observed in Phys. Rev. B 66, 153409-1-4 (2002) by R. I. Dzhioev et al. and Phys. Solid State 40, 2024-2030 
(1998) by V. P. Kochereshko et al.

5. I. A. Merkulov, Al. L. Efros, and M. Rosen, Phys. Rev. B 65, 205309-1-8 (2002). 